\title{
Low energy dark current collimation system in single-pass linacs
}

\author{
S. Bettoni, ${ }^{*}$ P. Craievich, M. Pedrozzi, M. Schaer, and L. Stingelin \\ Paul Scherrer Institut, 5232 Villigen, Switzerland
}

(Received 12 April 2017; published 21 February 2018; corrected 8 May 2018)

\begin{abstract}
The dark current emitted from a surface of a radio frequency cavity may be a severe issue for the activation and the protection of the components of linear accelerators, if this current is lost in an uncontrolled manner. For a single-pass linac based on a photo-injector, we studied the possibility of using a collimator installed at low energy (below $10 \mathrm{MeV}$ ) to dump the maximum fraction of the dark current before it is transported along the linac. We developed and experimentally verified an emission and tracking model that we used to study and optimize the dark current mitigation at SwissFEL test facility. We optimized a collimator, which is expected to reduce by two orders of magnitude the transport of the dark current to the first compressor. We have also verified the effects of wakefield excited by the beam itself passing through the collimator at such a low energy, comparing the results of beam-based measurements with an analytical model.
\end{abstract}

DOI: 10.1103/PhysRevAccelBeams.21.023401

\section{INTRODUCTION}

The electron sources commonly used in electron linear accelerators can be classified according to the emission process: thermionic emission, where the thermally excited electrons overcome the binding potential of the cathode material, and photoemission, where the electrons are extracted by a laser irradiating the cathode surface. In the latter scheme a high field at the gun is required to mitigate the space charge effects in the low beam energy section. This is of extreme importance to achieve the high brightness electron beams required for an efficient Free Electron Laser (FEL). Due to the high field of the radio frequency (rf) gun of the photo-injectors, not only the photocurrent (nominal beam used to run the accelerator), but also unwanted electrons (dark current) are parasitically emerging from the surface during the entire gun rf pulse length due to the field emission effect. The dynamics of a fraction of the dark current is similar to that of the nominal beam and, consequently, matched to the acceptance of the accelerator (optics and energy). This fraction can therefore be efficiently transported down to the higher energy sections, with a growing radiation dose associated with its loss. Similarly to the gun, all the structures may be sources of dark current, but the charges generated in the downstream sections are typically lost almost immediately after the emission because of the phase mismatch at the

*simona.bettoni@psi.ch

Published by the American Physical Society under the terms of the Creative Commons Attribution 4.0 International license. Further distribution of this work must maintain attribution to the author(s) and the published article's title, journal citation, and DOI. entrance of the other rf structures, the energy acceptance limitations in the dispersive sections, and the mismatch of the optics. This behavior has already been verified in previous studies [1], so in this work we neglect the contribution of the accelerating cavities downstream of the gun to the dark current. Several aspects contribute to the dark current emission from the gun. A smaller rf pulse length, typically associated with a smaller filling time of the cavity, produces less dark current. On the contrary a high surface field is associated to the emission of more dark current. The transport of the dark current out of the gun may also be strongly influenced by the transverse field distribution in the gun. All these aspects must be considered in the evaluation of the dark current.

Several studies and measurement campaigns have been done to understand the dark current emission and transport with the aim of minimizing the impact of its loss on the machine activation. A fast kicker may be used to cut the dark current tail after the main bunch, keeping this latter unaffected [2]. This technique is essential when operating with long rf pulses and bunch trains, but requires a relatively complex and expensive implementation.

We present here an alternative and easy to implement collimation configuration, well suited for short rf pulses (of the order of 1-5 $\mu \mathrm{s}$ ). After a description of SwissFEL and the SwissFEL Injector Test Facility (SITF) [3], in Sec. II, we focus on the guns installed in the facility, in Sec. III. In Sec. IV A we present the measurements performed at the low energy section of SITF and the emission model. In the following Sec. V we present the transport model of the dark current and we describe its outcome compared with the measurements in the high energy section of SITF in Sec. IV. In Sec. VI we present the measurements done to benchmark the numerical model and to characterize the impact of a 


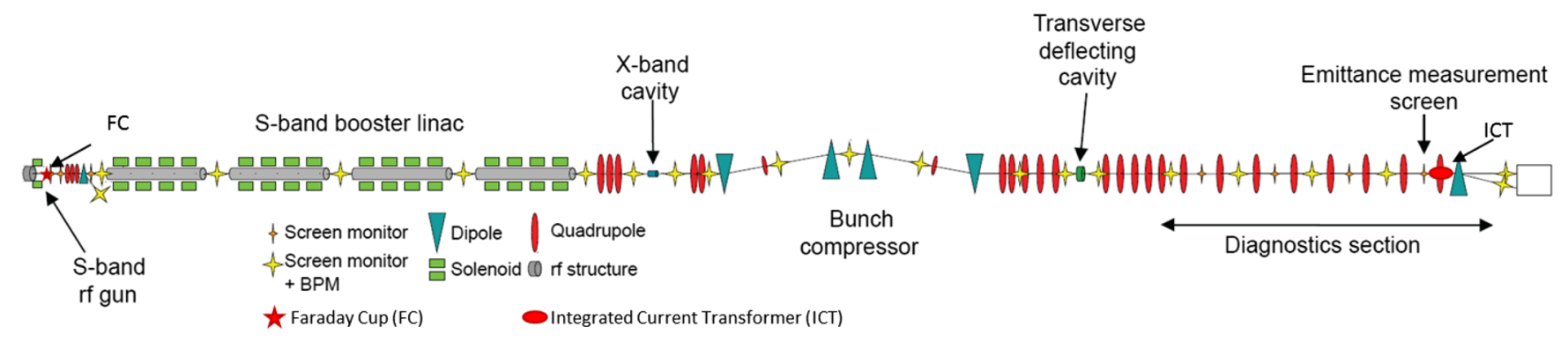

FIG. 1. SITF schematic layout (not in scale). The position of the faraday cup and the integrated current transformer used to measure the dark current are also indicated. The total length of the facility is about $60 \mathrm{~m}$.

collimator installed in the low energy section of the machine on the nominal SwissFEL beam. Finally in Sec. VII we discuss the effectiveness and the optimization of the method applied to SwissFEL [4].

\section{SwissFEL INJECTOR TEST FACILITY}

SwissFEL is the hard $\mathrm{x}$-ray FEL facility under commissioning at Paul Scherrer Institut (PSI). This machine, initially equipped with two undulator lines, will produce hard $\mathrm{x}$-rays for wavelengths ranging from 0.1 to $0.7 \mathrm{~nm}$, and soft x-rays from 0.65 to $5 \mathrm{~nm}$. The commissioning of the machine started in Summer 2016 and the first pilot user experiments are expected by the end of 2017. The SwissFEL Injector Test Facility (SITF) operated at PSI from March 2010 to October 2014, allowed for intensive and detailed beam dynamic studies and component developments toward SwissFEL. The schematic layout of the facility is shown in Fig. 1. The electron source is a laser driven S band (3 GHz) rf gun. Until April 2014 electrons were produced with an rf gun originally built for the CLIC test facility 2 at CERN, [5] kindly lent to PSI. In May 2014 the PSI-built SwissFEL rf gun [6] replaced the first device to allow for a higher $100 \mathrm{~Hz}$ repetition rate of SwissFEL compared to the $10 \mathrm{~Hz}$ of SITF. In both layouts the electrons were extracted by a laser pulse from an exchangeable copper cathode (at later stages in some cases coated with cesium telluride). From 2013 onwards the exchange of cathodes was facilitated by a load-lock chamber mounted onto the rf gun. Immediately after the gun, a movable solenoid magnet provides initial focusing and is used to optimize the emittance. In the drift between the gun and the first accelerating section a spectrometer is used to optimize the gradient and the phase of the gun with respect to the arrival time of the laser at the cathode. The main accelerating section consists of four S-band traveling-wave structures boosting the beam energy to a maximum value of $270 \mathrm{MeV}$. Each of the structures is surrounded by solenoids for symmetric transverse focusing at relatively low energy. Each solenoid is $75 \mathrm{~cm}$ long and provides magnetic fields up to $100 \mathrm{mT}$. The two downstream S-band structures are used to boost the beam energy and to impose the bunch energy chirp necessary for the longitudinal compression in the magnetic four dipoles chicane. Since the energy chirp generated in this way results in a curved longitudinal phase-space distribution, a fourth-harmonic cavity (X-band, at $12 \mathrm{GHz}$ frequency) is operated upstream of the bunch compressor to linearize the beam longitudinal phase space and the final compression. In nominal operation mode this cavity is set in anti-crest, inducing a net energy loss, bringing the final energy down to typically about $200 \mathrm{MeV}$. After the bunch compressor the beam enters an extensive diagnostic section, where the full 6D phase space may be characterized.

The SITF lattice and many components are very similar to those of the SwissFEL injector. The main differences are the number of S-band cavities upstream of the bunch compressor (six in SwissFEL instead of four in SITF), the laser heater in SwissFEL between the second and the third cavity, and the beam pipe aperture at the low energy section (internal diameter of $16 \mathrm{~mm}$ at SwissFEL and $38 \mathrm{~mm}$ at SITF). Except for the aperture difference, taken into account in the simulations, the differences of the lattice do not significantly affect the dark current transport, dominated by the gun electric field distribution and the distance between the cathode and the first focusing and accelerating elements.

\section{RF GUNS}

The first commissioning phases of SITF relied on the CTF2 gun No. V (referred as CTF2 gun for the rest of the paper), a 2.6-cell gun developed for high-current, multibunch operation at the CLIC test facility (CTF2) [5]. The general geometry of this gun is depicted in Fig. 2, together with electric field amplitudes in the vertical and horizontal sections. The unique feature of this gun compared to other designs is the large diameter of the first half cell, where the TM020-like resonance is used as the main accelerating mode. This gun provides an on-axis peak electric field of $100 \mathrm{MV} / \mathrm{m}$, with $88 \mathrm{MV} / \mathrm{m}$ at the cathode corresponding to a rf power of $21 \mathrm{MW}$ and a pulse length of $2 \mu$ s full length. This gun has a maximum repetition rate of $10 \mathrm{~Hz}$, limited by the cooling system. A new rf gun (referred as SwissFEL gun for the rest of the paper) was designed and manufactured in house to fulfill the operational requirements of 


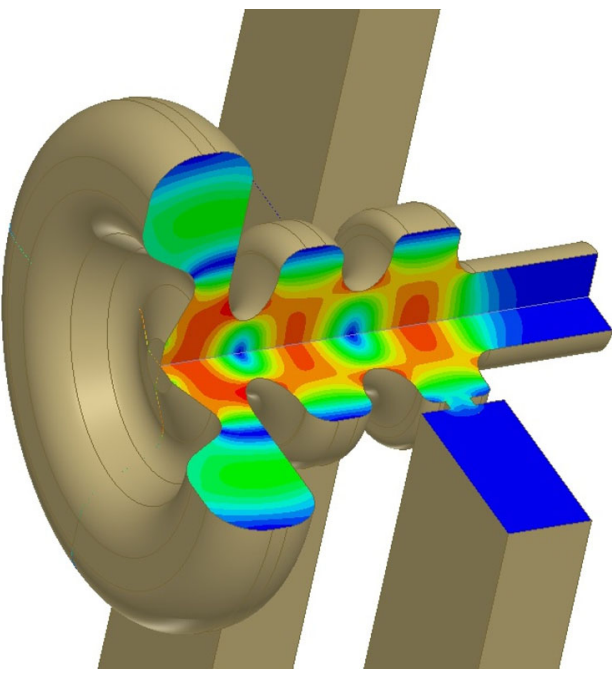

FIG. 2. Geometry of the CTF2 gun and amplitude of the electric field in the horizontal and vertical section.

SwissFEL, in particular high reliability at a higher repetition rate of $100 \mathrm{~Hz}$. Figure 4 shows the 3D drawing of the SwissFEL gun. The geometry is inspired by the 2.5 -cell PHIN gun [7,8], and adopts some mechanical design aspects of the LCLS gun $[9,10]$. Figure 4 also shows the 3D drawing of the gun backplane where the cathode is installed. The design is based on 2.6 cells operating with a near-perfect rotationally symmetric $\pi$-mode. The middle cell is coupled to two rectangular waveguides, symmetrically arranged to cancel the dipolar component of the field. The racetrack interior shape of this cell is optimized to minimize the quadrupolar field components. The gun provides an on-axis peak electric field of $100 \mathrm{MV} / \mathrm{m}$. Figure 3 shows the comparison of the on-axis longitudinal component of the electric field obtained from electromagnetic simulations for theCTF 2 and SwissFEL gun, respectively. In contrast to the CTF2 gun, the location of the on-axis peak field coincides with the cathode.

The backplanes of both the CTF2 and the SwissFEL gun feature circular openings where cathode plugs can be inserted. Figure 5 shows a standard polycristalline copper cathode plug (left) and cathodes (right) where a disc of

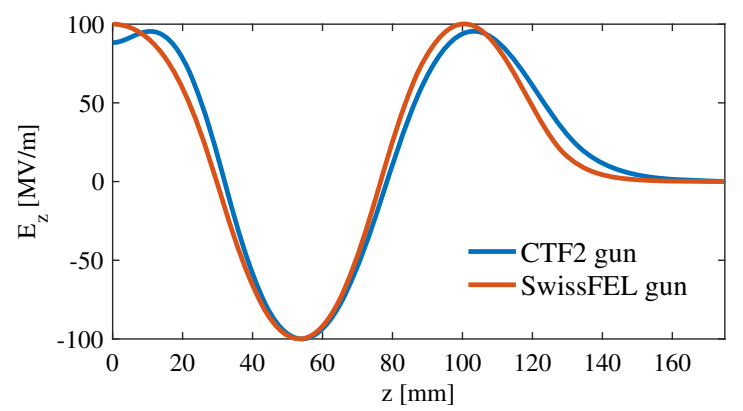

FIG. 3. Normalized on-axis longitudinal electric field profiles for the CTF2 and SwissFEL rf guns.

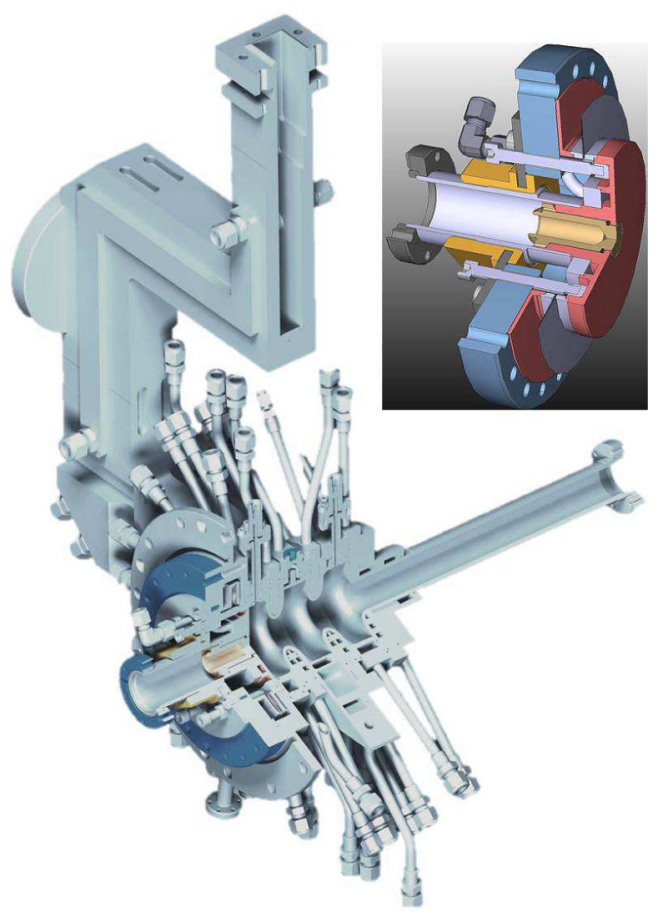

FIG. 4. Cutaway 3D views of the SwissFEL gun. Details of the backplane and the cathode plug are also visible in the upper right figure.

$10 \mathrm{~mm}$ diameter of $\mathrm{Cs}_{2} \mathrm{Te}$ has been deposited on the front surface.

A load-lock system [11] allows a relatively easy exchange of photocathodes in the two guns, giving the possibility of testing different materials, copper and the semiconductor $\mathrm{Cs}_{2} \mathrm{Te}$ (about two orders of magnitude higher quantum efficiency compared to copper), and repeating the measurements on several photocathodes and in this way verifying the reproducibility of the results. Copper cathode surfaces were diamond turned to an average roughness of a few nanometers [12]. For the semiconductor cathode the deposition procedure foresees $15 \mathrm{~nm}$ of tellurium and $25 \mathrm{~nm}$ of cesium, which are successively evaporated directly on the copper cathodes. As shown in Fig. 6 interferometry analysis has shown that the average surface roughness, $R_{a}$, is increased from $\approx 3 \mathrm{~nm}$ for pure copper to $\approx 15 \mathrm{~nm}$ once $\mathrm{Cs}_{2}$ Te is deposited.

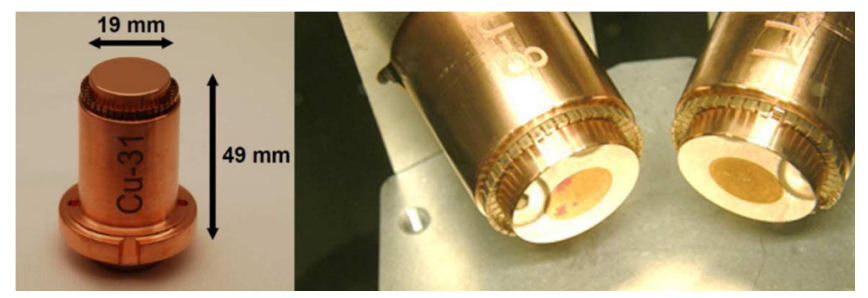

FIG. 5. Standard polycristalline copper cathode plug (left) and cathodes (right) where a disc of $10 \mathrm{~mm}$ diameter of $\mathrm{Cs}_{2} \mathrm{Te}$ has been deposited on the front surface. Both types were used at SITF. 


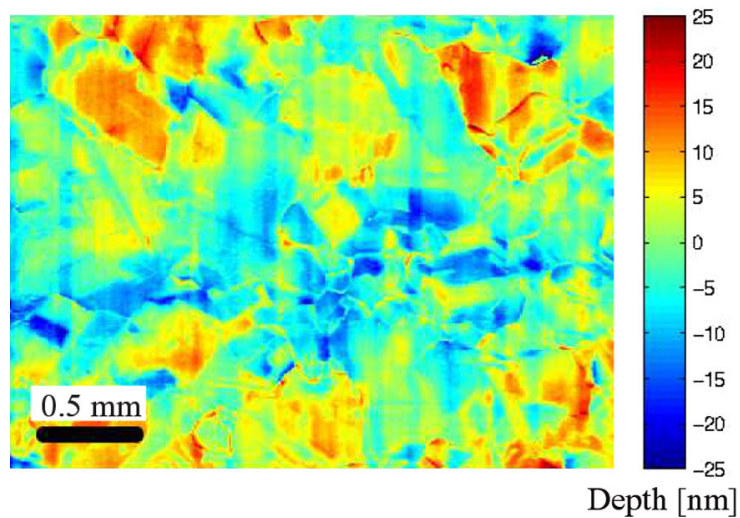

FIG. 6. Interferometric roughness measurement of cathode surface. Evaluated $R_{a}=5.2 \mathrm{~nm}$.

Figure 7 shows the electric field configuration in the interface area between the cathode plug and the backplane for the CTF2 and SwissFEL guns obtained from Superfish [13] simulations.

A larger fraction of particles emitted off-axis is lost in the SwissFEL gun compared to the CTF2case, due to the radial component of the electric field, which focuses the beam in the latter. Figure 8 shows the trajectory of particles emitted from the cathode plane tracked with AsTRA [14] at a time
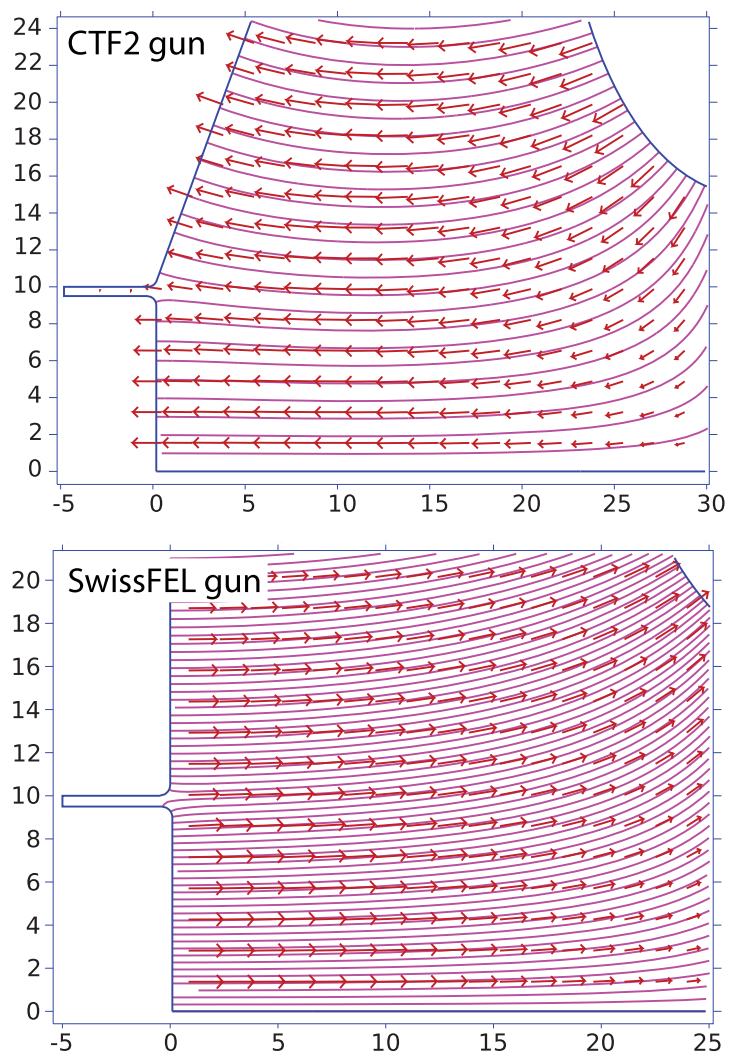

FIG. 7. Electric field pattern of the CTF2 and the SwissFEL gun obtained from numerical simulation. The axes are in $\mathrm{mm}$.
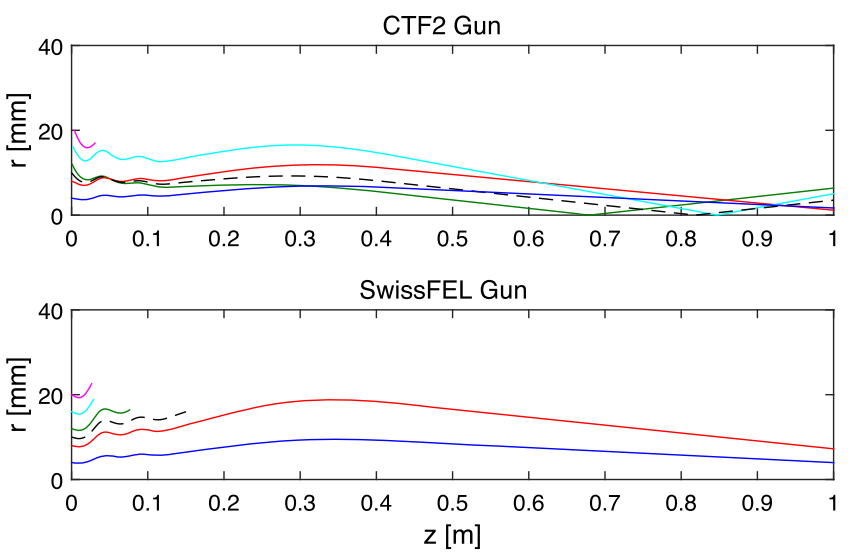

FIG. 8. Trajectories of particles emitted at distance $r=4,8,10$, $12,16,20 \mathrm{~mm}$ from the center of the cathode. Each line corresponds to a different distance. The emission is at the operating phase of the gun corresponding to the center of the emission time of the nominal beam. The dashed line corresponds to a starting position of $r=10 \mathrm{~mm}$, where the gap of the cathode plug lies. The top plot refers to the CTF2 and the bottom one to the PSI gun, respectively.

corresponding to the operating phase of the nominal beam for the CTF2 and the SwissFEL gun. We have a similar picture for the phase corresponding to the maximum field on the surface of the guns, as shown in Fig. 9. Several counteracting effects contribute to the dark current emission and transport through the gun. The higher field on the cathode and the distribution of the radial field may increase the emission and the transport of the dark current, whereas the shorter if pulse length possible with a smaller filling time of the gun may reduce this quantity. The SwissFEL gun has a larger peak field at the cathode than the CTF2
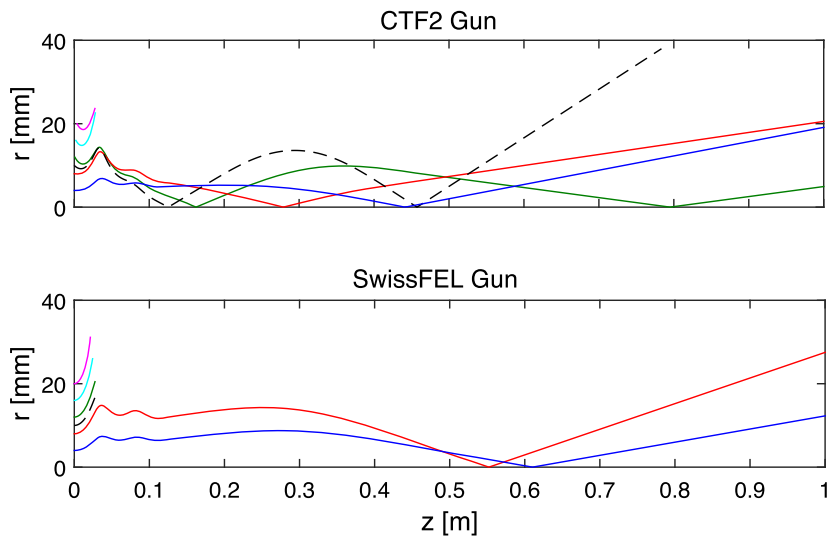

FIG. 9. Trajectories of particles emitted at distance $r=4,8,10$, 12, 16, $20 \mathrm{~mm}$ from the center of the cathode. Each line corresponds to a different distance. The emission is at the time corresponding to the maximum field of the gun. The dashed line corresponds to a starting position of $r=10 \mathrm{~mm}$, where the gap of the cathode plug lies. The top plot refers to the CTF2 and the bottom one to the PSI gun, respectively. 
gun, a smaller time constant (805 ns and $473 \mathrm{~ns}$ for the CTF2 and the SwissFEL gun, respectively) and less transverse focusing. The time constant refers to the rise time of the electromagnetic field inside the cavity. All these effects are considered to determine the dark current transported down to the first section of the machine.

\section{MEASUREMENTS AND ANALYSIS OF DARK CURRENT AT THE SITF}

We measured the dark current at two locations along the SITF: between the gun and the first accelerating cavity (low energy section), and at the end of the machine (high energy section). We present the measurements done using the different rf guns installed during the operation of SITF. The measurements in the low energy section, between the exit of the gun and the entrance of the first accelerating cavity (nominal beam energy of 7.1 MeV), described in Sec. IVA, are used to quantify the dark current extracted from the gun using different $\mathrm{rf}$ pulses and photocathode materials. The measurements at the end of the SITF (nominal beam energy of $230 \mathrm{MeV}$ ), described in Sec. IV B, aim to determine the fraction of the emitted dark current transported to the end of the machine, and benchmark the simulation model of the dark current transport. We foresee to dump the maximum possible fraction of dark current using a collimator installed upstream of the first accelerating cavity. As described in Sec. VIC, we measured the effect of the longitudinal and transverse wakefields of this device with the maximum SwissFEL bunch charge of $200 \mathrm{pC}$.

\section{A. Low energy section}

The first location where we measured the dark current at the SITF is at about $1 \mathrm{~m}$ from the cathode, where a
Faraday cup (FC) is installed. The rf parameters (phase and gradient) of the gun were optimized for nominal operation. We maximized the signal at the $\mathrm{FC}$ varying the field of the first solenoid downstream of the gun (typically lowering it by 30\%). Although this does not represent the dark current transmitted during nominal operation, it increases the signal-to-noise-ratio and corresponds to the maximum extracted dark current from the gun.

The field emission of electrons from a surface exposed to an electric field is a well-known phenomenon, described by the Fowler-Nordheim equation [15]. We express the current $I_{\mathrm{FN}}$ (in A) extracted for a given macroscopic electric field $E(t)$ on the cathode surface (in $\mathrm{V} / \mathrm{m}$ ) as $[16,17]$ :

$$
I_{\mathrm{FN}}(t)=I_{c} \times\left(\frac{E(t)}{E_{c}}\right)^{2} \times e^{-\frac{E_{c}}{E(t)}},
$$

with the functions:

$$
E_{c} \equiv 6.53 \times 10^{9} \frac{\phi^{1.5}}{\beta},
$$

and

$$
I_{c} \equiv A_{e} \times 65.67 \times 10^{-6} \times 10^{4.52 \cdot \phi^{-0.5}} \times \phi^{2},
$$

depending on the effective emitting area, $A_{e}\left(\right.$ in $\mathrm{nm}^{2}$ ), the work function of the material, $\phi$ (in $\mathrm{eV}$ ), and the dimensionless parameter $\beta$. The electric field $E(t)$, a function of the time in a single rf period and of the pulse length of the cavity (determined by the filling time $\tau$ of the structure and the target field), can be written as:

$$
E(t)= \begin{cases}E_{0}[1-\exp (-t / \tau)] \cos (\omega t) & \text { if } 0 \leq t<T_{\text {pulse }} \\ E\left(T_{\text {pulse }}\right) \times \exp \left[-\left(t-T_{\text {pulse }}\right) / \tau\right] \cos (\omega t) & \text { if } t \geq T_{\text {pulse }}\end{cases}
$$

where $T_{\text {pulse }}$ is the rf pulse length, $\omega$ is the rf angular frequency and $\tau$ is the time constant of the cavity. The field emission process was characterized measuring the integrated dark current, $Q$, as a function of the electric field at the cathode, and using the Fowler-Nordheim model to fit the parameters $I_{c}$ and $E_{c}$. Since these measurements were not performed at steady-state, the filling of the cavity described by Eq. (4) had to be included in the analysis of the measurements. We computed $Q$ by integrating Eq. (1) from $t=0$ to $2 T_{\text {pulse }}$ (for larger time the contribution to $Q$ is negligible), using Eq. (4) for the electric field:

$$
Q=\int_{0}^{2 T_{\text {pulse }}} I_{\mathrm{FN}}(t) d t
$$

Considering the electric field at cathode shown in Eq. (4) and the approximations $E_{0} \ll E_{c}$ and $\cos (\omega t) \approx$ $\left(1-\omega^{2} t^{2} / 2\right)$, we can write:

$$
Q=\sum_{j=1}^{N_{\mathrm{rf}}} \frac{T_{\mathrm{rf}}}{\sqrt{2 \pi}} I_{c}\left(\frac{E_{0, j}}{E_{c}}\right)^{2.5} \exp \left(-\frac{E_{c}}{E_{0, j}}\right)
$$

with $N_{\text {rf }}=2 T_{\text {pulse }} / T_{\text {rf }}, T_{\text {rf }}=2 \pi / \omega$ and with the approximation for the electric field in the interval $j T_{\text {rf }} \leq t \leq$ $(j+0.5) T_{\mathrm{rf}}$, which is

$$
E_{0, j}=E_{0}\left\{1-\frac{2 \tau}{T_{\mathrm{rf}}} \exp \left(-\frac{j T_{\mathrm{rf}}}{\tau}\right)\left[1-\exp \left(-\frac{T_{\mathrm{rf}}}{2 \tau}\right)\right]\right\},
$$



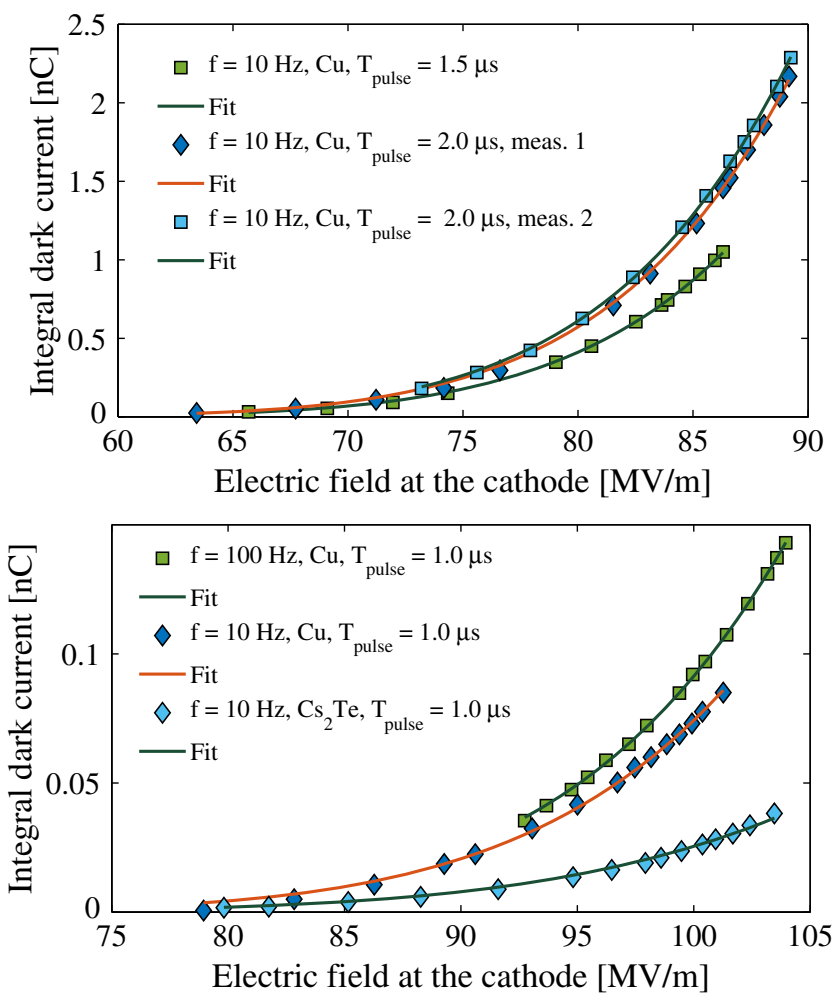

FIG. 10. Dark current versus the maximum electric field at the cathode measured for the CTF2 (top) and SwissFEL (bottom) guns. The repetition rate during the measurement, $f$, and the rf full pulse width, $T_{\text {pulse }}$, are also indicated.

for $0 \leq t<T_{\text {pulse }}$ and

$$
\begin{aligned}
E_{0, j}= & E_{0, N_{\mathrm{rf}}} \frac{2 \tau}{T_{\mathrm{rf}}} \exp \left(-\frac{\left(j-N_{\mathrm{rf}}\right) T_{\mathrm{rf}}}{\tau}\right) \\
& \times\left[1-\exp \left(-\frac{T_{\mathrm{rf}}}{2 \tau}\right)\right]
\end{aligned}
$$

for $t \geq T_{\text {pulse }}$. Figure 10 shows the measured $Q$ as a function of the field at the cathode for both rf guns operated at two different repetition rates and pulse widths and using two cathode materials. Table I shows the fitting parameters obtained from the measurements shown in Fig. 10. The coefficient of determination of the all data fit is higher that 0.999 and the root mean square error less than $1 \mathrm{pC}$. The measurements were reproducible as evidenced by the top plot in Fig. 10. Our measurements indicate a dependence of the dark current on the repetition rate (bottom plot of Fig. 10), which requires further investigations, but does not affect the conclusions of our study. We also observed a reduced dark current using a $\mathrm{Cs}_{2} \mathrm{Te}$ cathode compared to the copper, as shown in the bottom plot of Fig 10.

Figure 11 shows the envelope of the electric field at the center of the cathode and the dark current traces measured by the FC compared with those reconstructed assuming the $I_{c}$ and $E_{c}$ obtained from the measurements of the gun field amplitude. As showed in Fig. 11 the discrepancy between measured and expected pulses is less than $500 \mathrm{nA}$ and $20 \mathrm{nA}$ (residuals) for the CTF2 and the SwissFEL gun, respectively. Applying Eq. (2) and Eq. (3) we can derive the emitter area $A_{e}$ and the field enhancement factor $\beta$. Results are reported in Table I, for all the cases we assumed a work functions of $\phi=4.65 \mathrm{eV}$ and $\phi=4.1 \mathrm{eV}$ for copper and $\mathrm{Cs}_{2} \mathrm{Te}$ cathode material, respectively. Surface analysis of the cathodes, i.e. as in Fig. 6, did not show features that could explain such a large $\beta$ value. Our analysis of the experimental data of the emitted dark current together with the interferometric roughness measurements confirm the results in [17], i.e., that using a local low work function and reasonably low value of $\beta$ can better explain the surface analysis of the cathode. Figure 12 shows $\beta$ as a function of work function $\phi$ using the values of $E_{c}$ from Table I. It is worthwhile noting that it possible to fit the experimental data with different value of $\beta$ and $\phi$. Each point in the blue region on the plot represents a possible combination of field enhancement factor and local work function which match the data with a $95 \%$ confidence bound. The origin of local low work function at these sites is still unclear and should be further investigated. The dark current beam was also imaged on a $200 \mu \mathrm{m}$ thick YAG:Ce scintillating screen placed in the same housing of the FC. Figure 13 shows a comparison between images of the dark charges observed for the CTF2 and SwissFEL guns. Figure 13 (left) shows several field emitting points located along a ring, which

TABLE I. Parameters referring to the measurements shown in Fig. 10. $I_{c}$ and $E_{c}$ are fitting parameters with $95 \%$ confidence bounds also indicated in parenthesis. $A_{e}$ and $\beta$ are the emitter area and the field enhancement factor with $95 \%$ confidence bounds indicated in parenthesis.

\begin{tabular}{lccccccc}
\hline \hline Gun & \multicolumn{7}{c}{ rf width } \\
f $(\mathrm{Hz})$ & Cathode & $(\mu \mathrm{s})$ & $I_{c}(\mathrm{kA})$ & $E_{c}(\mathrm{MV} / \mathrm{m})$ & $A_{e}\left(\mu \mathrm{m}^{2}\right)$ & $\beta$ \\
\hline CTF2 & 10 & $\mathrm{Cu}$ & 1.5 & $17.7(15.1,23.0)$ & $773.8(773.6,774.1)$ & $0.1(0.08,0.13)$ & $84.6(84.5,84.6)$ \\
CTF2 & 10 & $\mathrm{Cu}$ & 2.0 & $17.7(14.2,22.1)$ & $780.8(780.4,781.3)$ & $0.1(0.08,0.12)$ & $83.9(83.8,83.9)$ \\
CTF2 & 10 & $\mathrm{Cu}$ & 2.0 & $17.7(14.5,21.6)$ & $780.3(779.5,782,1)$ & $0.1(0.08,0.12)$ & $83.9(83.7,84.0)$ \\
SwissFEL & 10 & $\mathrm{Cu}$ & 1.0 & $1.78(1.47,2.08)$ & $880.1(867.4,893.2)$ & $0.010(0.008,0.012)$ & $70.0(63.5,76.5)$ \\
SwissFEL & 100 & $\mathrm{Cu}$ & 1.0 & $1.27(0.44,2.11)$ & $870.6(825.0,921.7)$ & $0.007(0.003,0.011)$ & $75.2(71.0,79.4)$ \\
SwissFEL & 10 & $\mathrm{Cs}_{2} \mathrm{Te}$ & 1.0 & $0.12(0.02,0.23)$ & $774.3(708.7,853.4)$ & $0.0006(0.0001,0.0012)$ & $84.6(76.7,92.4)$ \\
\hline \hline
\end{tabular}



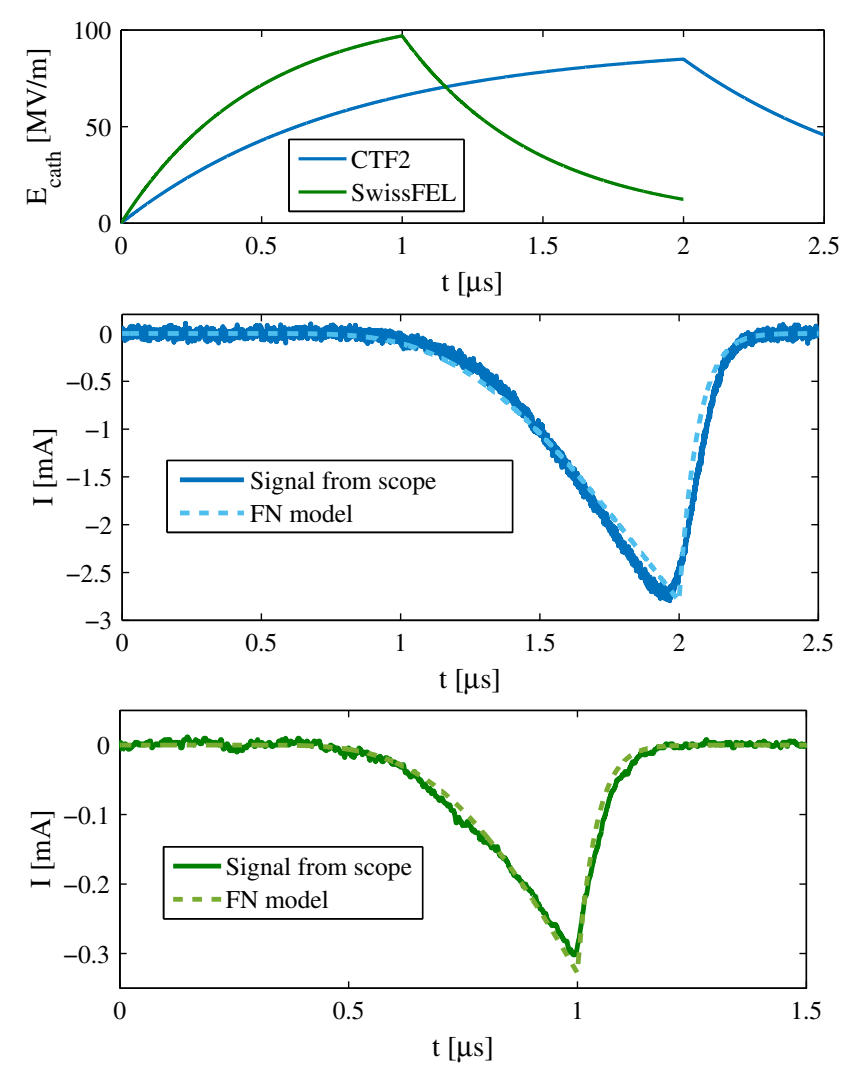

FIG. 11. Rf field amplitude of the SwissFEL and CTF2 gun (top plot), comparison of the FC measured trace and those obtained from the Fowler-Nordheim fit (dashed line) corresponding to the CTF2 (middle plot) and the SwissFEL (bottom plot) gun.

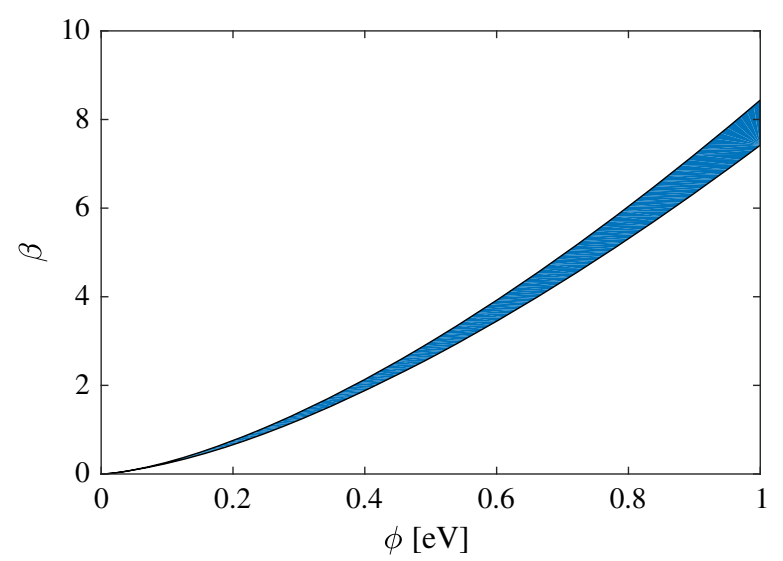

FIG. 12. $\quad \beta$ as a function of work function $\phi$ using the values of $E_{c}$ from table I. Each point in the blue region on the plot represents a possible combination of field enhancement factor and local work function which match the data with a $95 \%$ confidence bound.

may be identified with the aperture where the cathode plug and the spring for $\mathrm{rf}$ contact are located.

\section{B. High energy section}

We measured the transmission of the dark current downstream of the bunch compressor, using an integrated

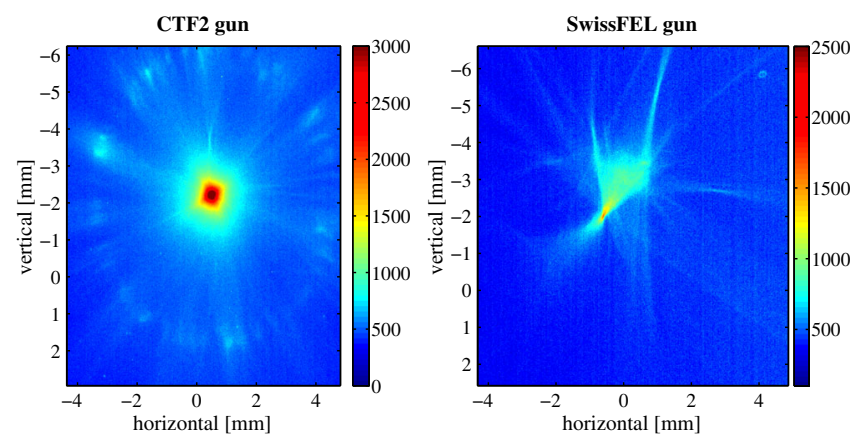

FIG. 13. Dark current beam imaged on a YAG:Ce screen near the gun exit for the CTF2 (left) and SwissFEL (right) gun. In both cases the electrons are focused with the gun solenoid.

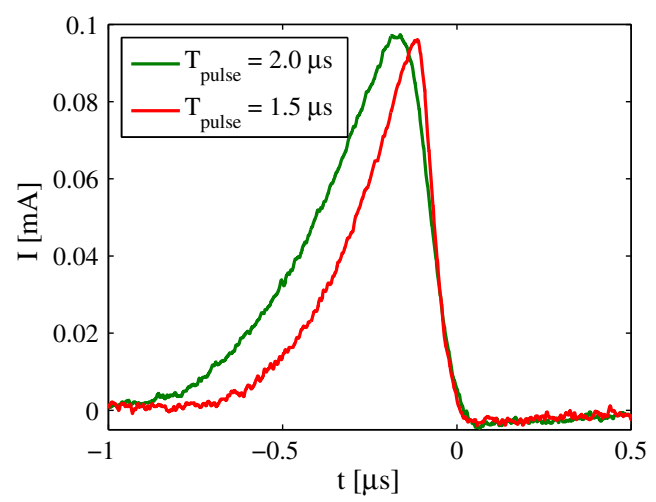

FIG. 14. Dark current measured by the ICT at $2 \mu \mathrm{s}$ and $1.5 \mu \mathrm{s}$ gun rf pulse lengths.

current transformer (ICT). We set the phases of all the S-band structures (energy gain per structure about $60 \mathrm{MeV}$ ) on-crest and the quadrupoles and the steerers to have a matched nominal beam with full transmission. We switched off the bunch compressor bends and we set the chicane in straight position. This allowed us to maximize the dark current transport, improving the signal-to-noise ratio at the ICT. This configuration gives an estimation of the total dark current transported down to the first bunch compressor, where in nominal operation losses are expected.

Figure 14 shows the typical dark current traces at the ICT for two initial pulse lengths. Table II reports the measured transmitted dark current using the considered pulse lengths.

Less than $2 \%$ of the charge measured at the low energy section is transported to the high energy section. The ratio of the transmitted charge from the low energy to the high energy section does not depend on the initial rf pulse length in the error bar range.

\section{TRANSPORT MODELING}

In this section we describe a model to study not only the emission, but also the propagation of the dark current along the machine, including focusing and acceleration. 
TABLE II. Field emitted charge measured at the low energy section of the machine for the CTF2 gun, and that transmitted to the end of the SITF in nominal conditions, but with the bunch compressor straight, assuming different rf pulse lengths. We report also the transmitted charge in the high energy section relative to that at the low energy region, and the ratio of the relative transmitted integrated current for the two considered pulse lengths.

\begin{tabular}{lccc}
\hline \hline & $Q_{\text {low }}(\mathrm{pC})$ & $Q_{\text {high }}(\mathrm{pC})$ & $Q_{\text {low }} / Q_{\text {high }}$ \\
\hline Pulse length $=1.5 \mu \mathrm{s}$ & $980 \pm 8$ & $19 \pm 2$ & $1.9 \% \pm 0.2 \%$ \\
Pulse length $=2.0 \mu \mathrm{s}$ & $1360 \pm 8$ & $27 \pm 1$ & $1.99 \% \pm 0.07 \%$ \\
$Q_{1.5 \mu \mathrm{s}} / Q_{2 \mu \mathrm{s}}$ & $72.1 \% \pm 0.7 \%$ & $70.4 \% \pm 0.8 \%$ & $1.02 \% \pm 0.01 \%$ \\
\hline \hline
\end{tabular}

Since the low energy section of the machine, where the charges are emitted, has a cylindrical symmetry ( $\mathrm{rf}$ cavities and surrounding solenoids), we developed a 2D model of the system using this fact. This approach allowed strongly reducing the computational time without losing information with respect to a fully 3D model. For all the calculations we generated the distribution according to the emission model previously described, and we tracked it using the numerical code ASTRA [14], assuming the machine parameters used for the experimental measurement and optimized for low emittance operation. We modified the ASTRA input file of the SITF with respect to the nominal case removing the calculation of space charge, negligible due to the very low charge density of the dark current, setting the phases of the rf cavities according to those used for the nominal beam, and we added an aperture to take into account the beam pipe. Furthermore, we used the $3 \mathrm{D}$ version of the code even if the problem has cylindrical symmetry to correctly compute the off-axis field in the gun far from the $\mathrm{rf}$ axis (in the 2D version ASTRA internally computes the transverse components of the electric field).

Let $z$ be the longitudinal coordinate of the linac and $r$ the distance from this axis, we emit the particles from any point $(z, r)$ along the surface of the gun for each time $t$ along a single rf period using Eq. (1). Due to the large number of $\mathrm{rf}$ periods in a pulse (several thousands in our cases), it would have been unpractical for computational time reasons to generate the real distribution using all of them along the full rf pulse length. Instead we preferred to discretize the rf pulse length in several sub-distributions of the length of a single rf bucket along the full rf pulse length. Each of these pulses is characterized by a different peak field determined according to Eq. (4). We computed for each of these distributions the fraction of the transmitted particles to the end of the second accelerating cavity. The result is shown in the top plot of Fig. 15. For our layout we can conclude that only less than $20 \%$ of the dark current emitted at time corresponding to a peak field smaller than $60 \mathrm{MV} / \mathrm{m}$ will be transmitted to the high energy section of the injector. The bottom plot of Fig. 15 shows the expected transmitted fraction of dark current normalized to that corresponding to $2 \mu \mathrm{s}$ rf pulse length as a function of the gun full rf pulse length. The model predicts a reduction of about $20 \%$ for a pulse shortening from $2.0 \mu \mathrm{s}$ to $1.5 \mu \mathrm{s}$, similarly to what was measured at the SITF (see Table II).

The length of the simulated dark current pulse at high energy shrinks with respect to that at low energy, due to the losses of the low energy tail mainly coming from the rising slope of the rf pulse and charges emitted far from the cathode axis, as shown in Fig. 16. This is also in agreement with the experimental observations at the SITF (see Fig. 14).
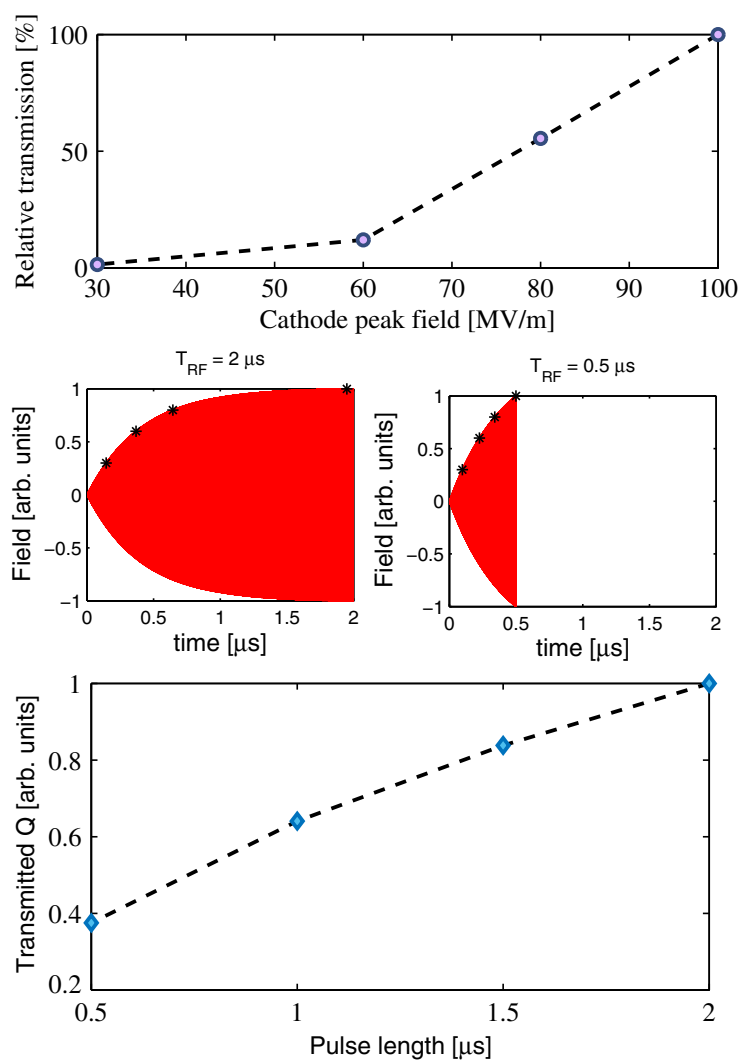

FIG. 15. Top plot: transmission to the end of the second accelerating cavity of the dark current as a function of the gun peak field. Middle plots: rf pulse assumed in the simulations assuming different pulse lengths. The buckets considered are also indicated. Bottom plot: expected transmission of the dark current to the end of the second accelerating cavity as a function of the rf pulse length normalized to the case corresponding to $2 \mu$ s rf pulse length. 

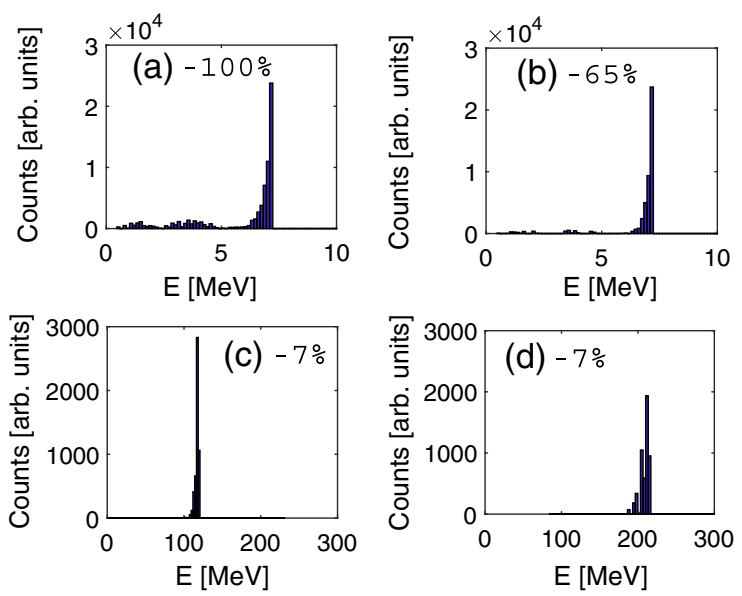

FIG. 16. Simulated energy spectrum of the dark current at (a) the location of the Faraday cup, (b) the entrance of the first accelerating cavity, (c) the exit of the second structure and (d) the end of the fourth structure. The percentage of the number macroparticles transmitted normalized to those at the FC is also shown. The rf pulse length assumed is $2 \mu \mathrm{s}$.

For completeness of the information a similar approach is also reported in $[18,19]$.

\section{THE DARK CURRENT MITIGATION EXPERIMENTAL STUDIES AT SITF}

A possible way to reduce the dose associated with the loss of dark current is to intercept the particles in the low energy section of the machine, between the gun and the first accelerating cavity, using a collimator. The best location for this device is the section upstream of the first accelerating cavity, where the nominal beam energy is $7.1 \mathrm{MeV}$. The dose associated with the controlled loss of dark current at this location would give less activation than what would be generated at higher energies along the machine.

We installed at SITF a sled with holes with different diameters with three goals: benchmark the simulation model, experimentally verify the effect of both longitudinal and transverse geometric wakefields excited by the nominal beam passing through the collimator, and reduce the dark current in SITF.

\section{A. Collimation at the SITF}

We installed a copper sled with different diameter holes, shown in Fig. 17. The thickness of the sled is $1.5 \mathrm{~cm}$, the height $20 \mathrm{~cm}$ and the width about $4 \mathrm{~cm}$, to ensure that the energy transported by the dark current and the nominal beam can be safely absorbed in case both are entirely lost on the sled. The sled is mounted on a vertical feed-through to allow selecting the different apertures and for beam-based studies.

One of the goals of these studies is to determine the impact of the excited wakefield on the nominal SwissFEL beam. We installed the sled at the SITF at $2.25 \mathrm{~m}$ from the cathode, where the nominal beam size $(\sigma=0.6 \mathrm{~mm})$ is

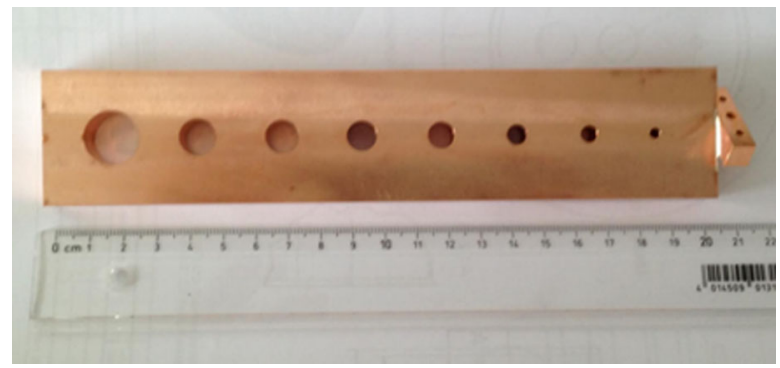

FIG. 17. Collimator array installed at SITF.

similar to that corresponding to the $200 \mathrm{pC}$ bunch charge of SwissFEL where the optimal position of the collimator is, as demonstrated in Sec. VII. We changed the diameter of the holes of the sled from $3 \mathrm{~mm}$ (slightly less than $3 \sigma$ ) up to $16 \mathrm{~mm}$ (diameter of the beam pipe aperture in SwissFEL) to perform these experiments. In this way we could study the effect of the wakefield on the beam, even if this is not the optimal position to collimate the dark current at the SITF.

\section{B. Benchmark of the simulation model}

We compared the measured dark current transmission at the end of SITF versus the collimator aperture with the simulations expectations using the CTF2 gun to validate the simulation model. Figure 18 shows the signals read by the ICT corresponding to different apertures of the collimator. We repeated the measurements in a time window of six months. The maximum deviations are below $10 \%$, even for the smallest apertures of the sled. This confirms the good degree of confidence in the measurement procedure. In Fig. 19 we compare the measured dark current transmission to the model predictions. The transmission is normalized to the charge at the end of SITF when the biggest collimator hole is inserted in the beam pipe (corresponding to the $16 \mathrm{~mm}$ diameter case). We considered the agreement between the model and the measurements satisfactory enough to justify its use to optimize the low energy collimation for SwissFEL.

\section{Effects of the collimator on the nominal beam}

The nominal beam passing through any cross section change may excite transverse and longitudinal geometric

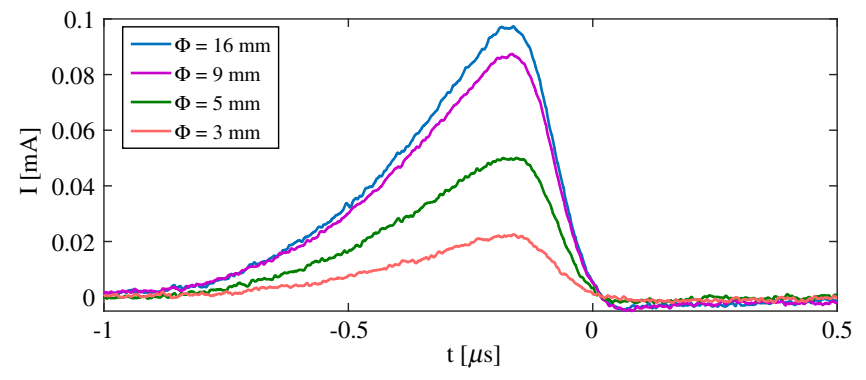

FIG. 18. ICT signals at the end of SITF using several aperture diameters, $\Phi$, of the collimator. 


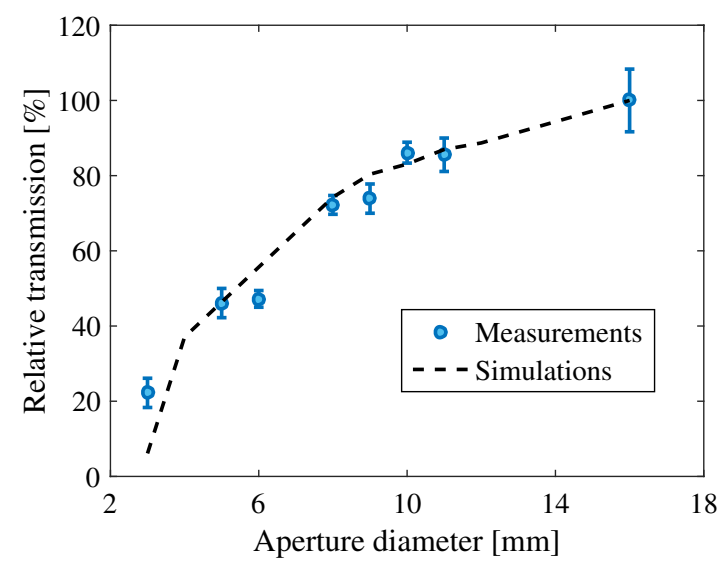

FIG. 19. Measured field emitted charge at the end of SITF as a function of the collimator aperture compared with the simulations. Each integrated current is normalized to the transmitted charge with the largest collimator aperture $(16 \mathrm{~mm})$.

wakefields, depending on the ratio of the apertures. The nominal beam during the measurements presented in the following is $2.7 \mathrm{ps}$ rms Gaussian pulse, $200 \mathrm{pC}$ charge (maximum for SwissFEL). Before including the collimator upstream of the first accelerating cavity in SwissFEL we verified the effect of these wakefields using the nominal maximum charge beam in SITF. We did not find any calculation or measurements of the wakefields for beam energy below $10 \mathrm{MeV}$ in the literature. We overestimated the effect of the wakefields, since we performed the measurements when a larger beam pipe (diameter of $38 \mathrm{~mm}$ ) was installed at SITF, whereas SwissFEL will use a beam pipe with a twice smaller radius. We measured the slice energy spread and the slice emittance along the bunch to check the impact of the longitudinal wakefield, and the beam projected emittance and the kick factor to characterize the effect of the transverse wakefield on the nominal SwissFEL beam.

We measured the slice energy spread of the nominal bunch by imaging the beam streaked using a transverse deflecting cavity (TDC) on a screen downstream of the last dipole upstream of the dump. The measurements reported in Fig. 20 do not indicate any significant increase of the slice energy spread within the resolution limit of our system ( $80 \mathrm{keV}$ at the time of these measurements).

We measured the projected emittance as a function of the diameter of the collimator by quadrupole scans. We did not observe any significant perturbation of the bunch introduced by the collimator for any of the available sled aperture diameters, as shown in Fig. 21.

The measurement of slice emittance versus collimator aperture, reported in Fig. 22, did not show any emittance degradation for any of the aperture of the collimator as well.

From these measurements we can therefore conclude that the longitudinal geometric wakefield induced by the low energy collimator with an aperture down to $3 \mathrm{~mm}$ is not expected to significantly degrade the maximum charge

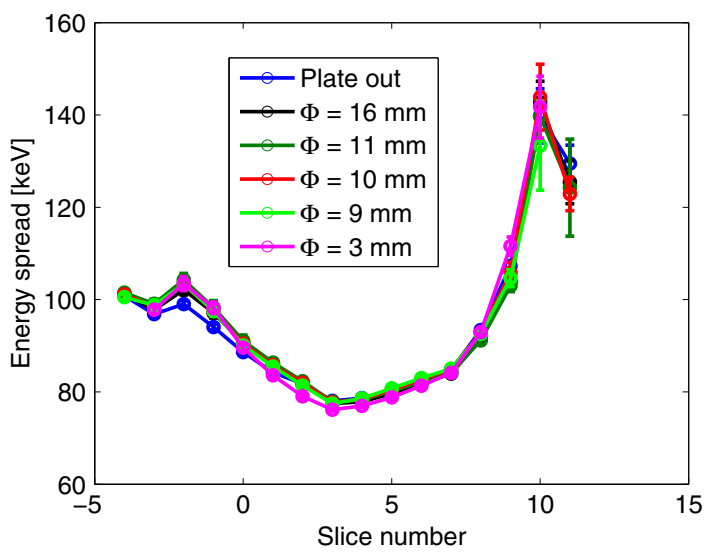

FIG. 20. Measured slice energy spread along the bunch for several collimator apertures compared to the case with the collimator removed from the beam path ("Plate out"). The full bunch length is divided in 15 parts of constant length.

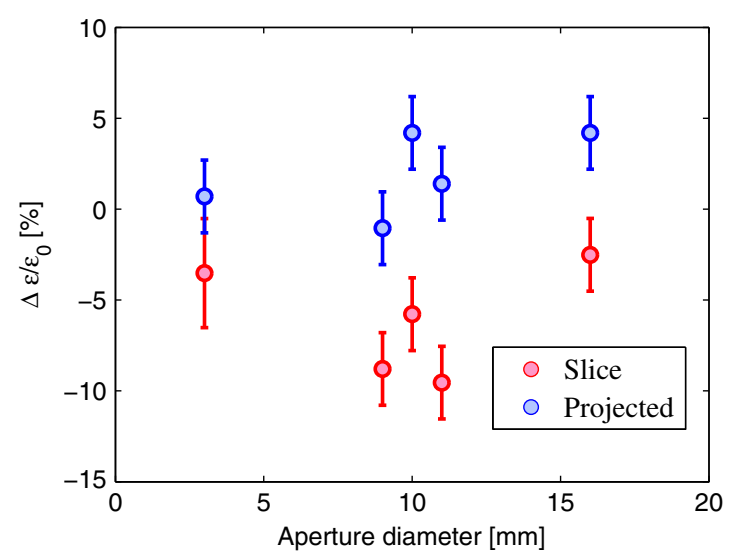

FIG. 21. Measured projected emittance increase versus the hole diameter of the collimator normalized to the case with the collimator removed from the beam path. The slice emittance of the central slice along the longitudinal coordinate of the bunch is reported for comparison to give an idea of the jitter of the machine.

SwissFEL beam quality, even if installed at a bunch energy of $7.1 \mathrm{MeV}$.

A transverse wakefield model for the collimator is useful to estimate the emittance growth for different apertures and beam parameters. Depending on the bunch length the wakefields can be inductive $[20,21]$ or diffractive. For the diffractive regime analytical formulas for the dipole kick factor exist in the limits of short [22] and long [23] collimators. The short range transverse wakefield is analyzed by moving the collimator plate vertically and measuring the centroid position $\left\langle y_{c}\right\rangle$ of the electron beam passing the aperture as a function of the displacement $\Delta y$ between the beam axis and the collimator center on a downstream YAG screen. Figure 23 shows the measured centroid position versus the displacement for a $3 \mathrm{~mm}$ collimator aperture. According to the theory, the centroid 


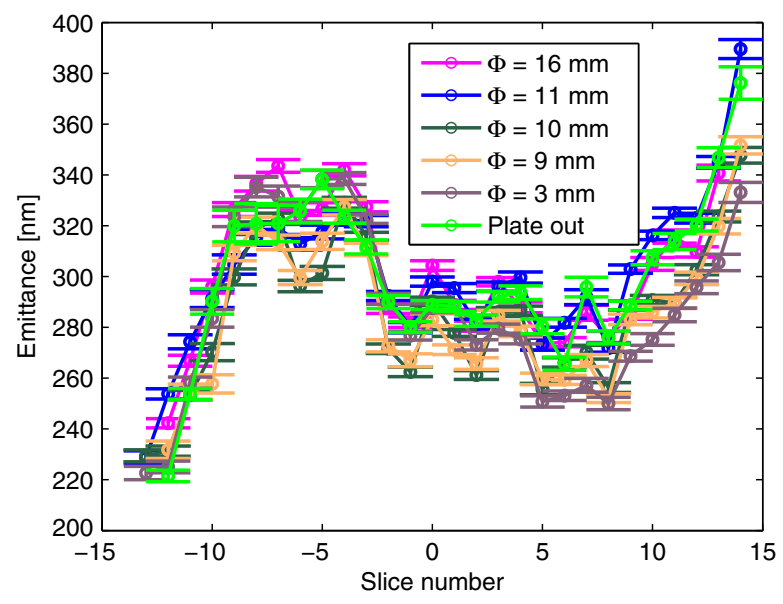

FIG. 22. Measured slice emittance along the bunch for the several diameter holes of the low energy collimator. The full bunch length is divided in 15 parts of constant size (not a constant charge per slice).

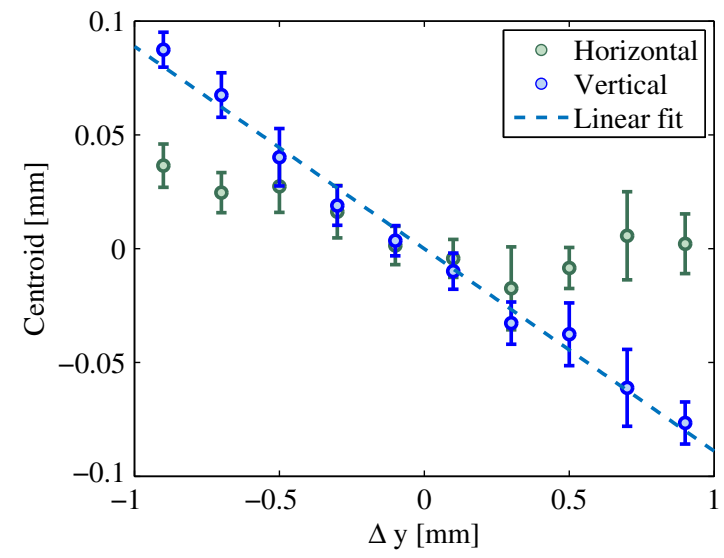

FIG. 23. Measured vertical position of the centroid of the electron beam passing the $3 \mathrm{~mm}$ diameter aperture of the collimator versus the displacement of the collimator axis with respect to the bunch on a downstream YAG screen. The horizontal centroid is shown to give an idea of the machine jitter.

position shift can be expressed as a function of the kick factor, $K_{\perp}$, as:

$$
\left\langle y_{c}\right\rangle=\frac{Q L}{E} K_{\perp} \cdot \Delta y
$$

where $E$ and $Q$ are the beam energy and charge, respectively, and $L$ is the distance between the collimator and the downstream YAG screen $(L=0.486 \mathrm{~m})$. The measurements were performed with a beam energy of $7.1 \mathrm{MeV}$, a Gaussian charge distribution with an rms bunch length of $0.95 \mathrm{~mm}$ and beam charge of $170 \mathrm{pC}$. The $K_{\perp}$ estimated from a linear fitting is $7.65 \pm 0.15 \mathrm{~V} /(\mathrm{pC} \mathrm{mm})$ with a coefficient of determination of the data fit higher than 0.98 . In case of a round and long collimator the analytical dipole kick factor is given by $[22,23]$ :
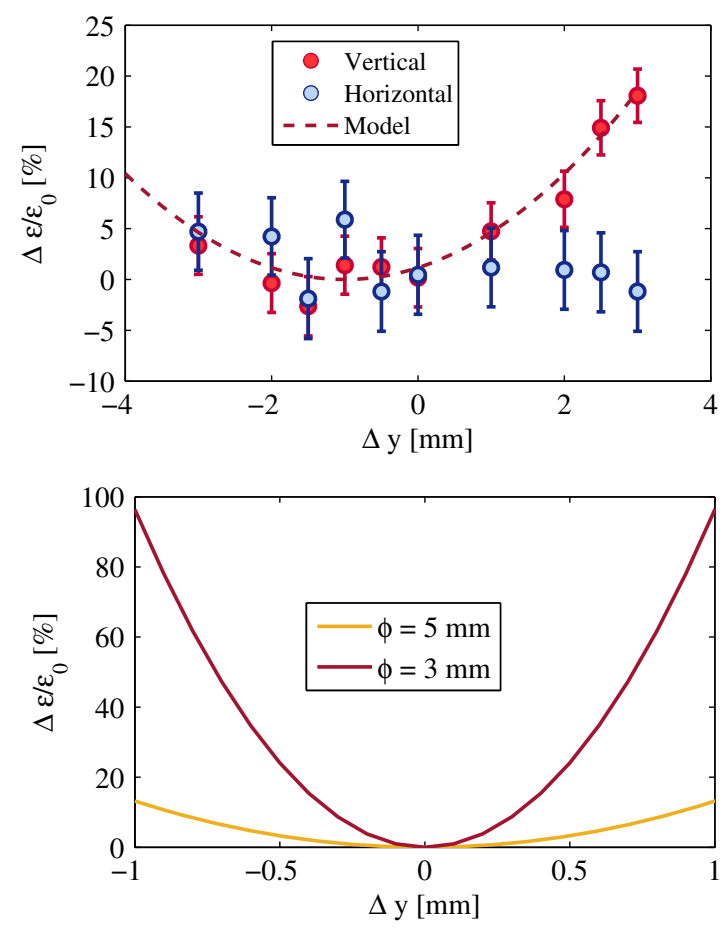

FIG. 24. Measured projected emittances with the $9 \mathrm{~mm}$ diameter hole as a function of the collimator offset making the beam passing through the $9 \mathrm{~mm}$ diameter hole of the collimator and comparison with Eq. (11) (top plot). Expectations from the model assuming several hole diameters (bottom plot).

$$
K_{\perp, \text { analy }}=\frac{Z_{0} c}{2 \pi} \cdot\left(\frac{1}{b_{2}^{2}}-\frac{1}{b_{1}^{2}}\right)
$$

with $b_{1}$ the radius of the beam pipe and $b_{2}$ the radius of the collimator aperture, $Z_{0}$ the vacuum impedance, and $c$ the vacuum light speed. With $b_{1}=19 \mathrm{~mm}$ and $b_{2}=1.5 \mathrm{~mm}$ the analytical kick factor is $7.95 \frac{\mathrm{V}}{\mathrm{pC} \cdot \mathrm{mm}}$, in agreement with that measured at SITF.

The emittance growth of the electron beam with Gaussian longitudinal distribution due to the transverse wakefield is given by [24]:

$$
\frac{\Delta \epsilon}{\epsilon_{0}} \sim \sqrt{1+\left(\frac{K_{\perp} Q \Delta y}{\sqrt{3} E}\right)^{2} \frac{\beta_{\text {Twiss }} \gamma}{\epsilon_{N}}},
$$

where $\beta_{\text {Twiss }}$ is the $\beta$ function at the location of the collimator, $\gamma$ is the relativistic factor, and $\epsilon_{N}$ is the normalized transverse emittance. Figure 24 (top) shows the relative emittance growth (dots) versus the beamcollimator offset $\Delta y$ for a collimator aperture of $9 \mathrm{~mm}$. In the same Fig. 24 the measurements are also compared with the estimated emittance increase calculated applying Eq. (11). Another method to estimate the dipole kick factor is to use a quadratic fit of the experimental data shown in Fig. 24. During the emittance measurements the beam orbit downstream of the collimator was fixed to avoid bunch 
deformations due to trajectory misalignment between the collimator and the screen for the emittance measurements. We measured an emittance blow-up for offsets larger than $\pm 1 \mathrm{~mm}$, as shown in Fig. 24 (top). Figure 24 (bottom) shows the emittance growth as a function of the transverse displacement for different collimator apertures obtained from the model in Eq. (11). The emittance growth is tolerable for all the collimator apertures if the transverse displacement is above the orbit alignment specifications routinely achieved in the machine.

In Sec. VII we will show that in SwissFEL a strong reduction of the dark current can be achieved with a $5 \mathrm{~mm}$ diameter hole collimator. This beam pipe restriction corresponds to about $8 \%$ projected emittance increase for a $1 \mathrm{~mm}$ offset at 7.1 MeV for the SwissFEL maximum bunch charge and with a larger beam pipe ratio (modified from the SITF to SwissFEL).

From these measurements we can therefore confirm the validity of the theory also for such a low beam energy, and that the beam with the highest beam charge foreseen in SwissFEL should not be degraded by the geometrical longitudinal and transverse wakefields excited by the bunch itself passing through the low energy collimator.

\section{DARK CURRENT MITIGATION AT SWISSFEL}

We studied the option to collimate the dark current in the low energy section of SwissFEL with the goal of propagating less than few tens of $\mathrm{pC}$ to the entrance of the first bunch compressor. We used the SITF to experimentally validate the emission and the tracking model of the dark current, and we did measurements to verify that there is no beam degradation due to a collimator installed in a low energy section. We used the same model to optimize the position and the aperture of the collimator to minimize the transmission of the dark current at SwissFEL. The position of the collimator in the SITF was determined by space constraints and it was chosen to have transverse sizes of the beam similar to those of the nominal SwissFEL bunch to properly study the possible beam degradation due to the wakefields. In SwissFEL we have more flexibility in changing the position of the components, since the machine has not been assembled yet.

According to the expectations from the simulations we should be able to drastically reduce the dark current transmission installing the collimator with $5 \mathrm{~mm}$ diameter aperture near the entrance of the first accelerating cavity. With this aperture we do not expect any degradation of the beam quality for offsets smaller than those already experimentally achieved at the SITF.

The two optimization parameters are the aperture of the collimator, limited by the possible degradation of the nominal beam due to the wakefields, and the position of the collimator, which must be compatible with the locations of the other components. Figure 25 shows the transmission of the dark current reaching the exit of the second

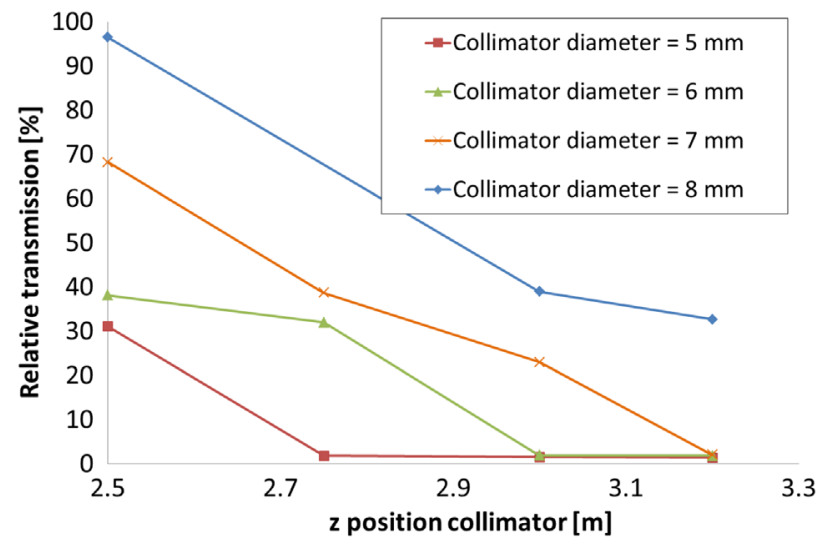

FIG. 25. Simulated transmission of the dark current at the exit of the second S-band structure at SwissFEL. The number of particles is normalized to those at the FC. The first accelerating structure is installed at $3.3 \mathrm{~m}$ from the cathode.

accelerating cavity normalized to that at the location of the FC as a function of these two parameters assuming the SwissFEL gun and layout. We limited the minimum collimator aperture to $5 \mathrm{~mm}$ considering this a safe margin for the dynamics of the nominal beam, and we decided to locate it as close as possible to the entrance of the first accelerating cavity, downstream of the waist of the low energy components of the dark current distribution, for which the first solenoid downstream of the gun is overfocusing the distribution. For the final design of SwissFEL we positioned a collimator of $1.5 \mathrm{~cm}$ thickness, $5 \mathrm{~mm}$ aperture at $3.2 \mathrm{~m}$ from the cathode plane. With this setup we expect to reduce the dark current transmission to less than $1 \%$, which, assuming the $80 \mathrm{pC}$ measured at the SITF with the SwissFEL gun corresponds to less than $1 \mathrm{pC}$ at the exit of the second cavity.

\section{CONCLUSIONS}

The dark current emitted from the surface of rf cavities may be a severe problem for the activation and the protection of the components of accelerators, if lost in an uncontrolled way. The emission of these charges from the surface of an rf cavity mainly depends on the surface intensity of the electric field and the properties of the surface, whereas the fraction of the particles transported out of the cavity is determined mainly by the transverse components of the electric field. We developed a model to study these phenomena, and we successfully applied it to explain the experimental behaviour of two rf guns installed in SITF: the latter characterized by larger field at the cathode but more focusing and the first with less field at the cathode but less focusing. We have also modeled the transport of the dark current from the exit of the gun toward the end of the machine. We experimentally verified this model by measuring the fraction of the dark current reaching the end of the machine. We have used the models 
to optimize a collimator to be installed between the gun and the first accelerating cavity to dump the maximum fraction of the dark current at low energy to minimize the dose associated to the loss of the particles. From this model we expect that a $1.5 \mathrm{~cm}$ thickness, $5 \mathrm{~mm}$ aperture collimator installed at the optimized position in SwissFEL will reduce the dark current transport down to the exit of the second accelerating cavity by two orders of magnitude. We have also verified both analytically and experimentally that the collimator installed at low energy does not degrade the SwissFEL nominal beam parameters.

\section{ACKNOWLEDGMENTS}

We would like to thank the SwissFEL beam diagnostics group, and in particular Rasmus Ischebeck and Markus Baldinger for the very prompt reactions to all our requests and the installation work done at SITF. We are also grateful to the PSI rf group and, in particular, to Ralf Menzel and Thomas Lippuner for supporting us in the rf tuning of the gun, and to all the other PSI expert groups and the SwissFEL team to the construction and operation of the SwissFEL Injector Test Facility. We are grateful to Thomas Schietinger for the careful proofreading of the paper.

[1] F. Le Pimpec, R. Zennaro, S. Reiche, and A. Adelmann, in Proceeding of the Free Electron Laser Conference, Nara, Japan, 2012 (JACoW, CERN, Geneva, 2012), MOPD36, p. 105.

[2] D. Lipka, W. Kleen, J. Lund-Nielsen, D. Noelle, S. Vilcins, and V. Vogel, in Proceedings of the 10th European Workshop on Beam Diagnostics and Instrumentation for Particle Accelerators, Hamburg, Germany, 2011 (Jacow, CERN, Geneva, 2011), WEOC03.

[3] T. Schietinger et al., Commissioning experience and beam physics measurements at the SwissFEL Injector Test Facility, Phys. Rev. Accel. Beams 19, 100702 (2016).

[4] R. Ganter, SwissFEL Conceptual Design Report, 2011.

[5] R. Bossart, H. Braun, M. Dehler, and J. C. Godot, A $3 \mathrm{GHz}$ photoelectron gun for high beam intensity, Nucl. Instrum. Methods Phys. Res., Sect. A 375, ABS7 (1996).

[6] J. Y. Raguin, M. Bopp, A. Citterio, and A. Scherer, in Proceedings of the Linear Accelerator Conference, Tel Aviv, Israel, 2012 (JACoW, CERN, Geneva, 2012), TUPLB01, p. 442.

[7] R. Losito et al., in Proceeding 10th European Particle Accelerator Conference, Edinburgh, Scotland, UK, 2006 (JACoW, CERN, Geneva, 2006), WEPLS059, p. 2517.

[8] R. Roux, Conception of photoinjectors for the ctf3 experiment, Int. J. Mod. Phys. A 22, 3925 (2007).
[9] L. Xiao, R. F. Boyce, D. H. Dowell, Z. Li, C. LimborgDeprey, and J. F. Schmerge, in Proceedings 21st Particle Accelerator Conference, Knoxville, TN, USA, 2005 (IEEE, Piscataway, NJ, 2005), pp. 3432-3434.

[10] D. H. Dowell et al., ICFA Beam Dynamics Newsletter 46, 162192 (2008).

[11] R. Ganter et al., in Proceeding 35th International FreeElectron Laser Conference, New York, NY, USA, 2013 (JACoW, CERN, Geneva, 2013), TUPSO21, p. 259.

[12] J. Bossert, R. Ganter, M. Schaer, and T. Schietinger, in Proceeding 36th International Free-Electron Laser Conference, Basel, Switzerland, 2014 (JACoW, CERN, Geneva, 2015), THP046, p. 832.

[13] Poisson Superfish, http://laacg.lanl.gov/laacg/services/serv_ codes.phtml.

[14] K. Floettmann, http://www.desy.de/ mpyflo/Astra_dokumen tation/ (2000).

[15] R. H. Fowler and L. Nordheim, Electron emission in intense electric fields, Proc. R. Soc. A 119, 173 (1928).

[16] R. Huang, D. Filippetto, C. F. Papadopoulos, H. Qian, F. Sannibale, and M. Zolotorev, Dark current studies on a normal-conducting high-brightness very-high-frequency electron gun operating in continuous wave mode, Phys. Rev. ST Accel. Beams 18, 013401 (2015).

[17] H. Chen, Y. Du, W. Gai, A. Grudiev, J. Hua, W. Huang, J. G. Power, E. E. Wisniewski, W. Wuensch, C. Tang, L. Yan, and Y. You, Surface-Emission Studies in a High-Field RF Gun based on Measurements of Field Emission and Schottky-Enabled Photoemission, Phys. Rev. Lett. 109, 204802 (2012).

[18] M. Santana Leitner, L. Ge, Z. Li, C. Xu, C. Adolphsen, M. Ross, and M. Carrasco, Studies of radiation fields of LCLS-II super conducting radio frequency cavities, Int. J. Mod. Phys. Conf. Ser. 44, 1660209 (2016).

[19] C. Xu et al., Proceedings of NAPAC 2016, Chicago, Illinois (USA) (JACoW, CERN, Geneva, 1996), MOPOB76, p. 235.

[20] K. Yokoya, CERN Technical Report No. CERN SL 90-88 (AP), 1990.

[21] G. Stupakov, Report No. SLAC-PUB-7086, 1996.

[22] I. Zagorodnov and K. Bane, in Proceedings of the European Particle Accelerator Conference, Edinburgh, Scotland, UK, 2006 (JACoW, CERN, Geneva, 2006), THPCH036, p. 2859.

[23] E. Gianfelice and L. Palumbo, Dipole impedance of a conducting cylindrical pipe with a discontinuous cross section, IEEE Trans. Nucl. Sci. 37, 1084 (1990).

[24] F. Zimmermann, K. L. F. Bane, and C. K. Ng, in Proceedings of the European Particle Accelerator Conference, Sitges, Spain, 1996 (JACoW, CERN, Geneva, 1996), TUP099G, p. 504.

Correction: Part of the image in the original Figure 7 contained an error and its replacement has been posted. 